\title{
Partial Cystectomy for Atypical Isolated Recurrence of Ovarian Adenocarcinoma - A Case Report and Literature Review
}

\author{
NICOLAE BACALBASA ${ }^{1}$ and IRINA BALESCU ${ }^{2}$ \\ ${ }^{1}$ Department of Obstetrics and Gynecology, \\ Carol Davila University of Medicine and Pharmacy, Bucharest, Romania; \\ ${ }^{2}$ Department of General Surgery, Ponderas Academic Hospital, Bucharest, Romania
}

\begin{abstract}
Background: Most cases with advanced-stage epithelial ovarian malignancies will experience recurrent disease at a certain moment of their evolution, even if maximal cytoreductive surgery has been performed at the moment of initial diagnosis. However, it seems that the best therapeutic strategy, in case of relapse, remains aggressive reresection, with complete cytoreduction being the most efficient way to improve survival. Materials and Methods: We present the case of a 55-year-old patient diagnosed with an isolated pelvic recurrence after stage IIIC surgically-treated ovarian cancer three years after primary cytoreduction. Results: Intraoperatively, the diagnosis of an isolated pelvic recurrence invading the urinary bladder was confirmed. The recurrent tumor was resected en bloc with partial cystectomy. The postoperative course was uneventful, while histopathological studies confirmed the presence of a poorly differentiated epithelial ovarian recurrent adenocarcinoma. At two year follow-up, the patient is free of any recurrent disease. Conclusion: Isolated pelvic recurrences after surgicallytreated ovarian adenocarcinomas can be safely removed and might improve survival.
\end{abstract}

Ovarian cancer remains one of the most common gynecologic malignancies affecting women worldwide and is commonly diagnosed in advanced stages of the disease when disseminated lesions are already present (1). In most of these cases, although the principles of complete cytoreductive surgery are applied, relapse will occur at a certain moment. The standard treatment for these patients

This article is freely accessible online.

Correspondence to: Nicolae Bacalbasa, Dimitrie Racovita Street, no. 2, Bucharest, Romania. Tel: +40 723540426, e-mail: nicolae_bacalbasa@ yahoo.ro

Key Words: Relapsed ovarian cancer, pelvic recurrence, partial cystectomy. has widely varied in the last decades; initially it has been considered that platinum-based chemotherapy should become the standard of care for 'platinum-sensitive' recurrent tumors, while the benefits of secondary cytoreductive surgery have been considered uncertain (2). However, this theory was discarded by studies demonstrating that debulking surgery is able to increase the biological efficacy of chemotherapeutic agents by removing all bulky tumors with poor vascular supply (3). The benefits of secondary cytoreduction at the time of relapse seem to be more evident in cases presenting good performance status, early stage at diagnosis, no residual disease after primary cytoreduction, association of adjuvant chemotherapy, longer disease-free survival, normal cancer antigen (CA)-125 levels, no ascites and isolated recurrences (4-10). The good postoperative outcome reported by patients presenting isolated relapse is mainly explained by the limited number of visceral resections required to obtain complete cytoreduction, transforming these cases in perfect candidates for secondary cytoreduction $(11,12)$. Unfortunately, it is well-known that this eventuality is a rare one as most cases present multiple recurrences necessitating an important visceral sacrifice both in the lower and upper abdomen (1315). The aim of this work is to present the case of a 55-yearold patient diagnosed with an atypical, isolated pelvic recurrence invading the urinary bladder removed by performing a partial cystectomy three years after initial diagnosis of ovarian cancer.

\section{Case Report}

A 55-year-old patient with a medical history of stage IIIC ovarian cancer presented in our service for pelvic pain and macroscopic hematuria. The patient had been submitted to cytoreductive surgery three years before; total hysterectomy with bilateral adnexectomy, pelvic, parietal bilateral peritonectomy, omentectomy, pelvic and para-aortic lymph node dissection. At that moment, an R0 resection was achieved, while the histopathological examination revealed 


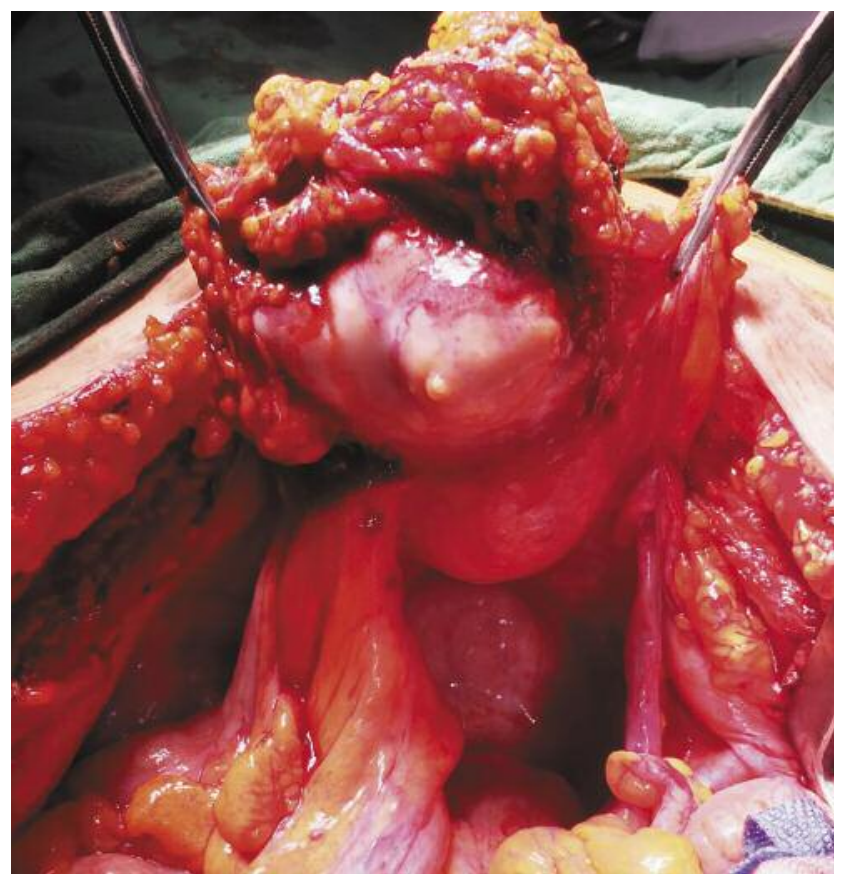

Figure 1. Initial aspect: Pelvic recurrence invading the urinary bladder and adherent to the sigmoid loop.

the presence of a medium to poorly differentiated epithelial serous ovarian adenocarcinoma. Postoperatively, the patient was submitted to six cycles of adjuvant platinum- and taxane-based chemotherapy, while the follow-up revealed no recurrent disease for the next two years and half. The imagistic studies performed at the current moment revealed the presence of an isolated tumor located on the posterior wall of the urinary bladder, in close connection with the sigmoid loop. The patient was submitted to cystoscopy, which confirmed the lesion that was biopsied. The histopathological examination confirmed the ovarian metastatic origin and, thus, the patient was re-submitted to surgery; intraoperatively, the presence of the isolated recurrence in the urinary bladder wall, adherent the sigmoid loop, was confirmed (Figure 1). The recurrent tumor was resected en bloc with partial cystectomy, while the digestive structures were successfully dissected and preserved. The parietal defect of the urinary bladder was closed throughout a two layer closure of 2-0 running, delayed absorbable, nonlocking stich (Figures 2-7); the transurethral Foley catheter was retrieved in the 21th postoperative day, after performing a computed tomography in order to confirm the intact repair of the urinary bladder. Since the postoperative course was uneventful, the patient was discharged in the fifth postoperative day. Histopathological examination confirmed the ovarian origin of the tumor, which was successfully

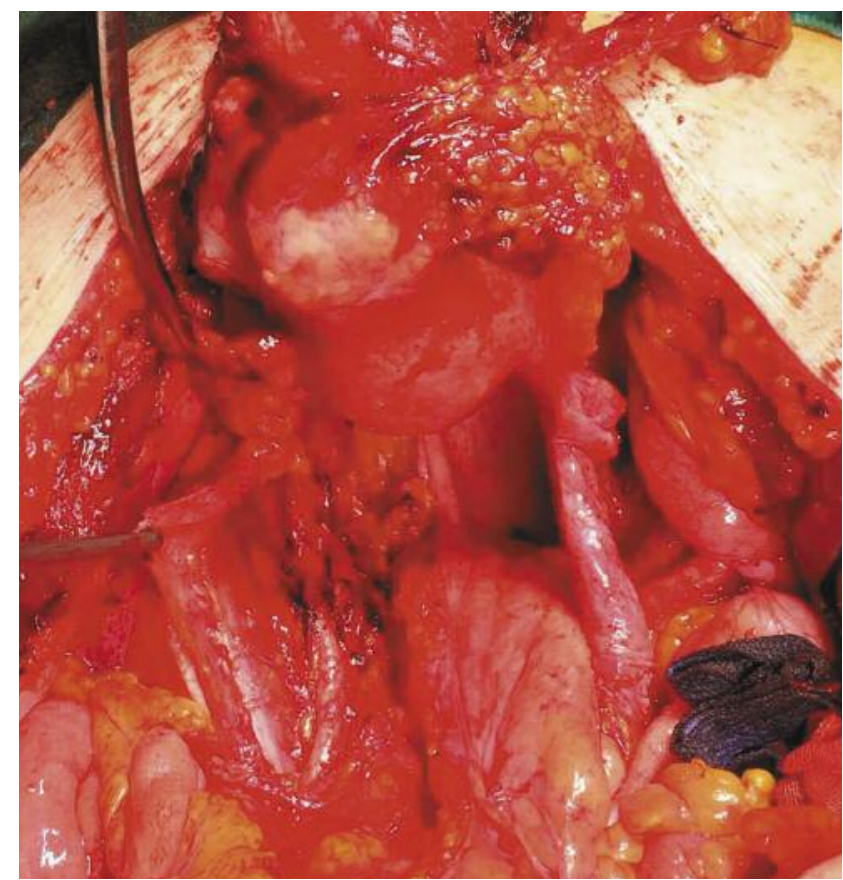

Figure 2. Tumor mobilization with rectosigmoid preservation.

removed with negative resection margins. At two years of follow-up, the patient is free of any local or distant recurrent disease.

\section{Discussion}

Cases presenting isolated recurrences with ovarian cancer origin are uncommon since most cases are exhibiting disseminated lesions at the time of diagnosis. Fortunately, this has been widely demonstrated to be a favorable prognostic factor, being associated with high rates of longterm survival whenever complete cytoreductive surgery is performed $(4,16,17)$. Moreover, among patients presenting localized relapsed, cases with lymph node recurrence seem to have the best overall prognosis (17). One of the largest studies conducted on the theme of the influence on survival of the anatomic site of relapse comes from Petrillo et al. demonstrating that cases with isolated lymph node recurrences experience a significantly higher overall survival versus cases with isolated peritoneal lesions or versus cases with isolated parenchymatous lesions, with median progression-free survival being 63 versus 41 versus 24 months (11). In the meantime, the presence of peritoneal carcinomatosis has been reported in up to $70 \%$ of patients with recurrent disease but failed to be a negative prognostic factor on the overall survival if complete debulking surgery 


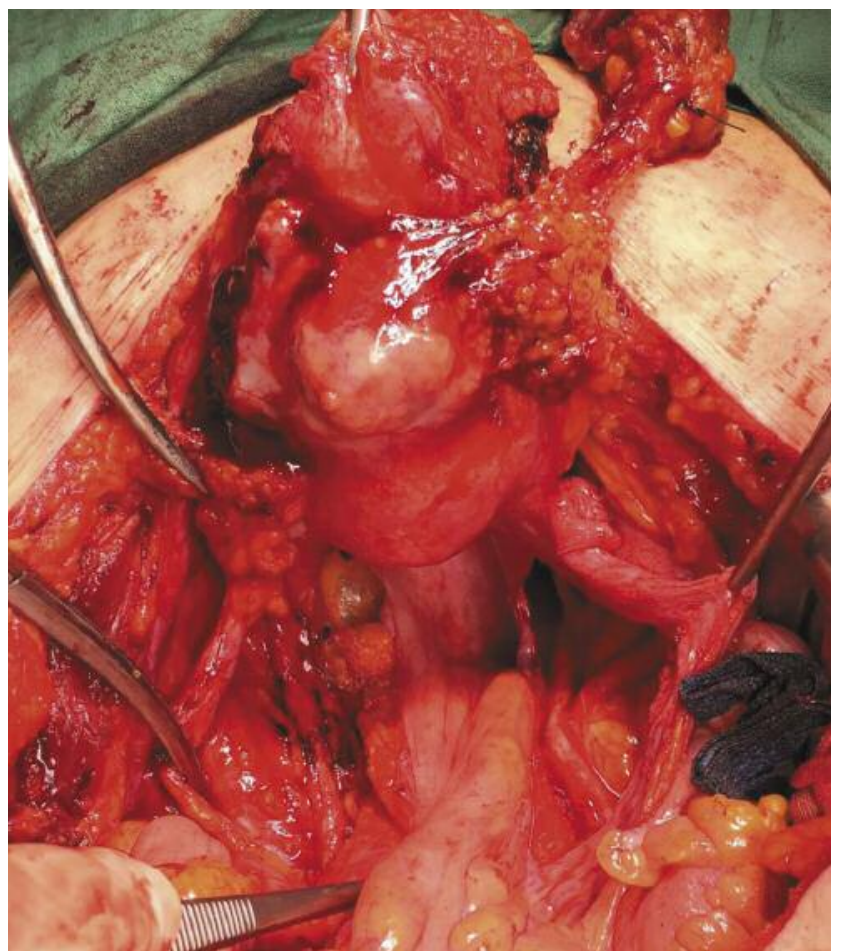

Figure 3. Dissection of the rectosigmoid loop and the two ureters.

to no residual disease is feasible (18). The benefits of complete cytoreductive surgery at the time of relapse have been best revealed by Bristow et al.'s meta-analysis, published in 2009, including 2,019 patients from 40 studies. The authors created a regression model demonstrating that the median overall survival time increased from 18 months to 48 months if the proportion of cases submitted to complete cytoreductive surgery increased from 0 to $100 \%$ (with each 10\% increase of the proportion of cases submitted to complete cytoreduction at the moment of the first relapse being associated with a 3-month increase of the median survival of the cohort) (19).

In order to determine the patients to potentially benefit most after secondary cytoreduction, Minaguchi et al. conducted a study on 80 cases submitted to secondary cytoreduction for ovarian cancer relapse; the authors studied the influence of four different favorable prognostic factors on the long-term outcome: treatment-free interval longer than 12 months, absence of distant metastases, presence of isolated lesions and 0 performance status. The authors demonstrated that cases achieving three or all of the four factors are the best candidates for secondary cytoreductive surgery (20). According to Minaguchi et al.'s theory, our patient was the perfect candidate for secondary cytoreductive surgery, with all four criteria being fulfilled.

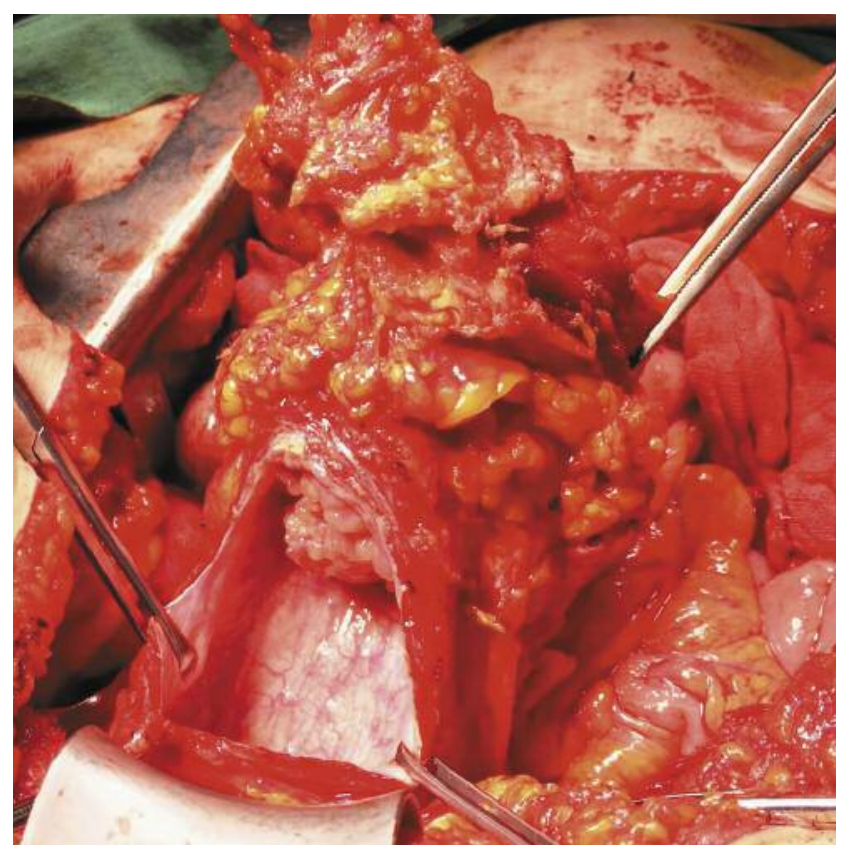

Figure 4. The aspect after urinary bladder transection; the pelvic recurrence invaded the urinary bladder mucosa.

Another particularity of our case is the location of the relapse in the urinary bladder requiring a partial cystectomy. The presence of an infiltrating tumor at this level necessitated partial urinary bladder resection with $1 \mathrm{~cm}$ resection macroscopic-free margin; however, this resection did not interfere with the functional outcome of the patient, with more than $30 \%$ of the urinary bladder capacity being preserved.

The feasibility of urinary tract resections, as part of debulking surgery for advanced stage ovarian cancer, was initially demonstrated by the study of Malviya et al.; the study included 22 patients submitted to surgery between 1983 and 1987. The most commonly performed urinary tract resections included partial cystectomy (in seven cases) followed by ureteroneocystostomy (in five cases), partial cystectomies with ureteroneocystostomy (in four cases) and uretero-ureteral anastomosis (in other four cases). The authors reported nine major and two minor complications that occurred in seven cases, with one patient being dead at six weeks postoperatively. When it comes to the long-term outcomes, Malviya et al. reported a mean survival period of 15.2 months for patients in whom complete cytoreduction had been achieved. The authors concluded that urinary tract resections are justified if complete debulking surgery is achievable with acceptable rates of postoperative morbidity (21). 


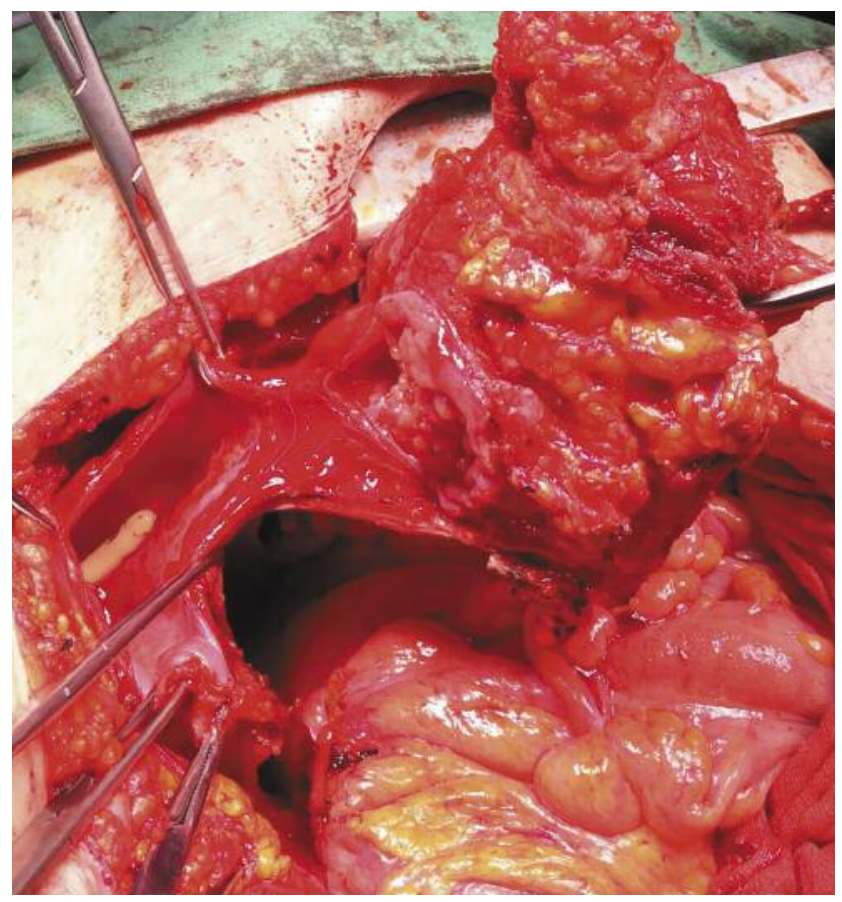

Figure 5. Performing partial cystectomy.

However, literature data regarding urinary tract resections as part of debulking surgery for advanced stage or relapsed ovarian cancer are scarce; most often these resections are presented as collateral surgical procedures for pelvic tumors with ovarian origin and rectosigmoid involvement (22-24).

In Park et al.'s study, the authors included 60 patients submitted to multiple visceral resections for advanced stage or relapsed ovarian cancer; among these cases, 14 patients were introduced in the current study at the moment of secondary cytoreductive surgery, with six of them necessitating urinary tract resections. The most commonly performed urinary tract resections at the time of secondary cytoreductive surgery included ureteral resection with uretero-ureteral anastomosis and partial cystectomy and did not associate any specific urinary complication (23). Another study that reported the association of urinary tract resections as part of debulking surgery for pelvic recurrences after surgically treated ovarian cancer comes from Bristow and colleagues encompassing 56 patients submitted to surgery at Kelly Gynecologic Service, Baltimore, MD, USA, and at the Division of Gynecologic Oncology of the European Institute of Oncology, Milan, Italy. Urinary tract resections consisted of segmental ureteral resections and partial cystectomies and were performed in eight cases. In all cases, the continuity of the urinary tract was reestablished by ureteroneocystostomy and psoas hitch with or without Boari flap and did not associate any urinary leaks (24).

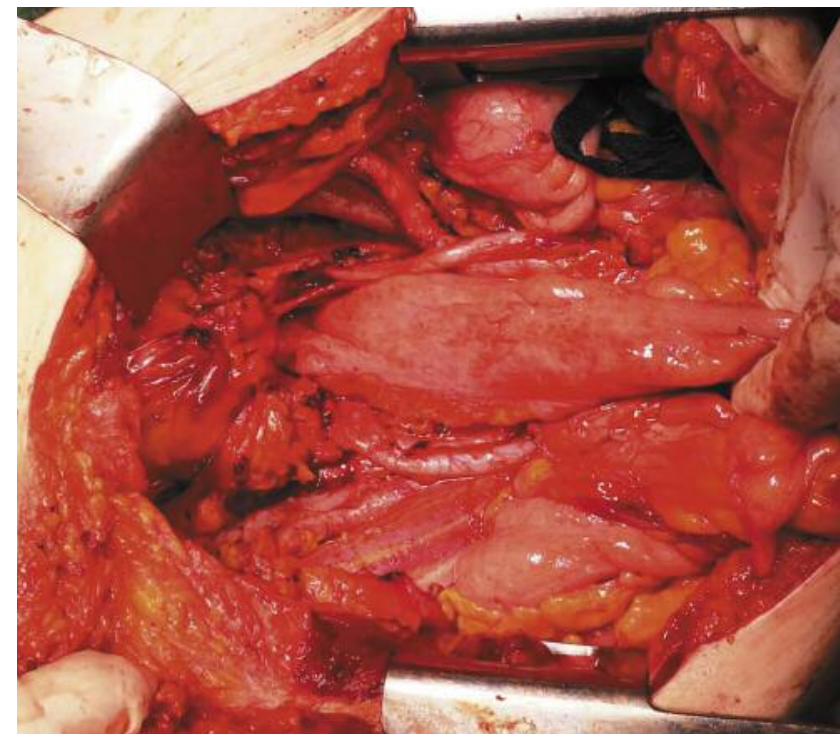

Figure 6. The final aspect after recurrent tumor resection en bloc with partial cystectomy. The continuity of the digestive tract was entirely preserved.

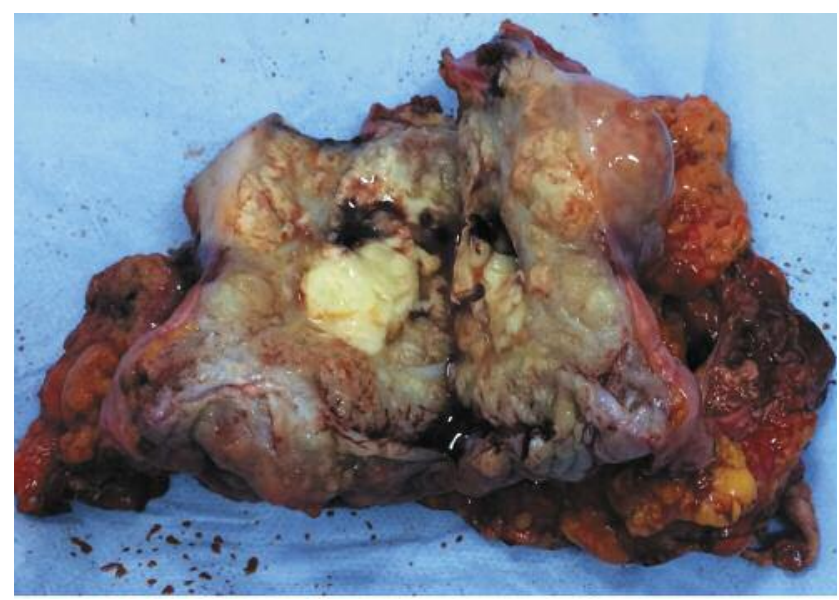

Figure 7. The specimen: The pelvic recurrence was resected en bloc with partial cystectomy.

\section{Conclusion}

Isolated pelvic recurrences after advanced-stage, surgicallytreated ovarian cancer are rare eventualities and associate a good long-term outcome in terms of disease-free and overall survival if cytoreductive surgery is performed. The particularity of our case consists of the limited pelvic visceral invasion of the recurrent tumor, imposing only a partial cystectomy and no digestive visceral sacrifice. In the meantime, the tumor location out of the urinary bladder 
trigone and the absence of any sign of ureteral ostium invasion pointed to a limited cystectomy with no need of ureteral resection or reimplantation.

\section{Acknowledgements}

This work was supported by a grant of the Romanian National Authority for Scientific Research and Innovation, CNCS UEFISCDI, project number PN-II-RU-TE-2014-4-2533.

\section{References}

1 Shih KK and Chi DS: Maximal cytoreductive effort in epithelial ovarian cancer surgery. J Gynecol Oncol 21: 75-80, 2010.

2 Lee CK, Lord S, Grunewald T, Gebski V, Hardy-Bessard AC, Sehouli J, Woie K, Heywood M, Schauer C, Vergote I, Scambia G, Ferrero A, Harter P, Pujade-Lauraine E and Friedlander M: Impact of secondary cytoreductive surgery on survival in patients with platinum sensitive recurrent ovarian cancer: Analysis of the CALYPSO trial. Gynecol Oncol 136: 18-24, 2015.

3 Boente MP, Chi DS and Hoskins WJ: The role of surgery in the management of ovarian cancer: primary and interval cytoreductive surgery. Semin Oncol 25: 326-334, 1998.

4 Zang RY, Li ZT, Tang J, Cheng X, Cai SM, Zhang ZY and Teng $\mathrm{NN}$ : Secondary cytoreductive surgery for patients with relapsed epithelial ovarian carcinoma: Who benefits? Cancer 100: 11521161, 2004.

5 Onda T, Yoshikawa H, Yasugi T, Yamada M, Matsumoto K and Taketani Y: Secondary cytoreductive surgery for recurrent epithelial ovarian carcinoma: Proposal for patients selection. $\mathrm{Br}$ J Cancer 92: 1026-1032, 2005.

6 Chi DS, McCaughty K, Diaz JP, Huh J, Schwabenbauer S, Hummer AJ, Venkatraman ES, Aghajanian C, Sonoda Y, AbuRustum NR and Barakat RR: Guidelines and selection criteria for secondary cytoreductive surgery in patients with recurrent, platinum-sensitive epithelial ovarian carcinoma. Cancer 106: 1933-1939, 2006.

7 Eisenhauer EA, Vermorken JB and van Glabbeke M: Predictors of response to subsequent chemotherapy in platinum pretreated ovarian cancer: A multivariate analysis of 704 patients [seecomments]. Ann Oncol 8: 963-968, 1997.

8 Bacalbasa N, Balescu I, Dima S, Herlea V, David L, Brasoveanu $\mathrm{V}$ and Popescu I: Initial incomplete surgery modifies prognosis in advanced ovarian cancer regardless of subsequent management. Anticancer Res 35: 2315-2320, 2015.

9 Bacalbasa N, Dima S, Balescu I, David L, Brasoveanu V and Popescu I: Results of Primary Cytoreductive Surgery in Advanced-stage Epithelial Ovarian Cancer: A Single-center Experience. Anticancer Res 35: 4099-4104, 2015.

10 Bacalbasa N, Balescu I, Dima S, Brasoveanu V and Popescu I: Hematogenous splenic metastases as an independent negative prognosis factor at the moment of primary cytoreduction in advanced stage epithelial ovarian cancer - A single center experience. Anticancer Res 35: 5649-5654, 2015.

11 Petrillo M, Fagotti A, Ferrandina G, Fanfani F, Costantini B, Vizzielli G, Pedone AL, Nero C, Margariti PA and Scambia G: Ovarian cancer patients with localized relapse: Clinical outcome and prognostic factors. Gynecol Oncol 131: 36-41, 2013.
12 Salani R, Santillan A, Zahurak ML, Giuntoli RL, Gardner GJ, Armstrong DK and Bristow RE: Secondary cytoreductive surgery for localized, recurrent epithelial ovarian cancer: Analysis of prognostic factors and survival outcome. Cancer 109: 685-691, 2007.

13 Bacalbasa N, Balescu I, Dima S, Brasoveanu V and Popescu I: Pancreatic resection as part of cytoreductive surgery in advanced-stage and recurrent epithelial ovarian cancer - A single-center experience. Anticancer Res 35: 4125-4129, 2015.

14 Bacalbasa N, Dima S, Brasoveanu V, David L, Balescu I, Purnichescu-Purtan R and Popescu I: Liver resection for ovarian cancer liver metastases as part of cytoreductive surgery is safe and may bring survival benefit. World J Surg Oncol 13: 235, 2015.

15 Bacalbasa N, Balescu I, Dima S and Popescu I: Long-term survivors after liver resection for ovarian cancer liver metastases. Anticancer Res 35: 6919-6923, 2015.

16 Munkarah A, Levenback C, Wolf JK, Bodurka-Bevers D, Tortolero-Luna G, Morris RT and Gershenson DM: Secondary cytoreductive surgery for localized intra-abdominal recurrences in epithelial ovarian cancer. Gynecol Oncol 81: 237-241, 2001.

17 Eisenkop SM, Friedman RL and Spirtos NM: The role of secondary cytoreductive surgery in the treatment of patients with recurrent epithelial ovarian carcinoma. Cancer 88: 144-153, 2000.

18 Harter P, Hahmann M, Lueck HJ, Poelcher M, Wimberger P, Ortmann O, Canzler U, Richter B, Wagner U, Hasenburg A, Burges A, Loibl S, Meier W, Huober J, Fink D, Schroeder W, Muenstedt K, Schmalfeldt B, Emons G and du BA: Surgery for recurrent ovarian cancer: Role of peritoneal carcinomatosis: Exploratory analysis of the DESKTOP I Trial about risk factors, surgical implications, and prognostic value of peritoneal carcinomatosis. Ann Surg Oncol 16: 1324-1330, 2009.

19 Bristow RE, Puri I and Chi DS: Cytoreductive surgery for recurrent ovarian cancer: A meta-analysis. Gynecol Oncol 112: 265-274, 2009.

20 Minaguchi T, Satoh T, Matsumoto K, Sakurai M, Ochi H, Onuki M, Oki A and Yoshikawa H: Proposal for selection criteria of secondary cytoreductive surgery in recurrent epithelial ovarian, tubal, and peritoneal cancers. Int J Clin Oncol 21: 573-579, 2016.

21 Malviya VK, Malone JM Jr. and Deppe G: Advanced ovarian cancer: Urinary tract resection as a part of cytoreductive surgery. Eur J Gynaecol Oncol 10: 69-72, 1989.

22 Plotti F, Montera R, Aloisi A, Scaletta G, Capriglione S, Luvero D, De Cicco NC, Basile S, Benedetti PP and Angioli R: Total rectosigmoidectomy versus partial rectal resection in primary debulking surgery for advanced ovarian cancer. Eur J Surg Oncol 42: 383-390, 2016.

23 Park JY, Seo SS, Kang S, Lee KB, Lim SY, Choi HS and Park SY: The benefits of low anterior en bloc resection as part of cytoreductive surgery for advanced primary and recurrent epithelial ovarian cancer patients outweigh morbidity concerns. Gynecol Oncol 103: 977-984, 2006.

24 Bristow RE, Peiretti M, Gerardi M, Zanagnolo V, Ueda S, DiazMontes T, Giuntoli RL and Maggioni A: Secondary cytoreductive surgery including rectosigmoid colectomy for recurrent ovarian cancer: Operative technique and clinical outcome. Gynecol Oncol 114: 173-177, 2009.

Received February 26, 2017

Revised March 17, 2017

Accepted March 20, 2017 